\title{
bFGF Polymorphism Is Associated with Disease Progression and Response to Chemotherapy in Multiple Myeloma Patients
}

\author{
TOMASZ WRÓBEL ${ }^{1 *}$, ALEKSANDRA BUTRYM ${ }^{2 *}$, PIOTR ŁACINA ${ }^{3}$, JUSTYNA RYBKA ${ }^{1}$, \\ KATARZYNA GĘBURA ${ }^{3}$, GRZEGORZ MAZUR ${ }^{4}$ and KATARZYNA BOGUNIA-KUBIK ${ }^{3,4}$ \\ ${ }^{1}$ Department of Haematology, Blood Neoplasms and Bone Marrow Transplantation, \\ Wroclaw Medical University, Wrocław, Poland; \\ ${ }^{2}$ Department of Physiology, Wroclaw Medical University, Wrocław, Poland; \\ ${ }^{3}$ Laboratory of Clinical Immunogenetics and Pharmacogenetics, Hirszfeld Institute of Immunology and \\ Experimental Therapy, Polish Academy of Sciences, Wrocław, Poland; \\ ${ }^{4}$ Department and Clinic of Internal and Occupational Diseases, \\ Hypertension and Clinical Oncology, Wroclaw Medical University, Wrocław, Poland
}

\begin{abstract}
Background/Aim: Vascular endothelial growth factor (VEGF) and basic fibroblast growth factor (bFGF) play an important role in the initiation of angiogenesis. We aimed to assess whether polymorphisms within the genes coding for these angiogenic activators (VEGF (rs3025039;C>T) and bFGF $(r s 308395 ; G>C))$ contribute to susceptibility and/or progression in multiple myeloma patients $(M M)$ and to chemotherapy. Patients and Methods: One hundred and thirtytwo patients with MM and 122 controls were genotyped for the VEGF and bFGF alleles by the PCR-RFLP technique. Genotyping results were compared regarding progression, risk of disease and response to treatment. Results: Patients in stage I-II disease (Durie-Salmon criteria) more frequently carried the bFGF $-921 G$ allele compared to patients in stage III $(p=0.053)$ and healthy controls $(O R=2.010, p=0.040)$. Progression after first-line chemotherapy was more frequent among patients carrying this variant $(p=0.022)$. Conclusion: Our results imply that the course of disease in MM patients is associated with a polymorphism within the bFGF gene.
\end{abstract}

Multiple myeloma is a plasma cell malignancy characterized by proliferation of clonal plasma cells in the bone marrow

\footnotetext{
*These Authors contributed equally to this study.

Correspondence to: Prof. Katarzyna Bogunia-Kubik, Hirszfeld Institute of Immunology and Experimental Therapy, Polish Academy of Sciences, Weigla 12, 53-114 Wroclaw, Poland. Tel: +48 71 3709960, Fax: +48 71 3371382, e-mail: bogunia@iitd.pan.wroc.pl

Key Words: VEGF gene polymorphism, $b F G F$ gene polymorphism, multiple myeloma, disease susceptibility, response to immunomodulating therapy.
}

and secretion of monoclonal immunoglobulins detectable in the serum and/or urine. The process of angiogenesis has been proven to play a crucial role in the progression of multiple myeloma. Imbalance between antiangiogenic and proangiogenic factors (among them FGF - fibroblast growth factor and VEGF - vascular endothelial growth factor) can lead to the so-called "angiogenic switch", triggering vascular phase of the disease and inducing the clonal expansion of myeloma cells $(1,2)$. Evidence that VEGF expression is elevated in multiple myeloma has given rise to the use of antiangiogenic drugs in clinical practice (3).

The human VEGF gene is located on chromosome 6p21.3 and is organized into 8 exons separated by 7 introns (4). Several single nucleotide polymorphisms (SNPs) have been described in the VEGF gene, including a $\mathrm{C}$ to $\mathrm{T}$ substitution at position 936 (rs3025039) within the 3'-untranslated region (3'UTR), that is related to VEGF protein production (5). VEGF plasma levels were significantly lower in carriers of the $936 \mathrm{~T}$ allele. Such observations could be attributed to the $936 \mathrm{C} / \mathrm{T}$ transition leading to loss of a potential binding sites for transcription factor AP-4 (activating enhancer binding protein 4).

The human $b F G F$ gene is located on chromosome 4 (6). Polymorphisms within the promoter region of the $b F G F$ gene may interfere with existing transcription factor binding sites or produce new binding sites and, therefore, influence $b F G F$ gene expression (7).

In our previous studies, genetic variants of the $V E G F$ and/or $b F G F$ genes were found to contribute to the development of haematological malignancies - chronic lymphocytic leukaemia (CLL) (8) and non-Hodgkin's lymphoma (NHL) (9). In patients with NHL, the presence of the VEGF $936 T$ allele was found to be associated significantly with worse prognosis of the disease, that was represented by a higher International 
Prognostic Index (3-4 vs. 0-2 and 4 vs. 0-3 score, $p<0.05$ ). The $b F G F-913 G$ variant was more frequently detected among NHL patients with aggressive histological subtype than those with indolent histological subtype and healthy individuals (9). In CLL, only a slight prevalence of the VEGF $\mathrm{T}$ variant was observed among patients as compared to healthy individuals, with a significant difference when high risk (stage III/IV) CLL patients were considered (8).

In the present study, a $C$ to $T$ substitution at position 936 within the 3'-untranslated region of the VEGF gene (rs3025039) and a $C$ to $G$ substitution at position -921 within the promoter region of the $b F G F$ gene (rs308395) were analyzed in order to determine whether the presence of these allelic variants was associated with susceptibility and progression of the disease in patients with multiple myeloma. Patient response to immunomodulating therapy was also studied.

\section{Patients and Methods}

Patients. One hundred and thirty-two patients aged 37-85 years (median age $=61$ ), diagnosed with multiple myeloma, were included into the study. There were 70 men and 62 women. In 15 cases diagnosis of multiple myeloma was proceeded by MGUS (monoclonal gammopathy of undetermined significance). 20 patients had renal insufficiency. Clinical characteristics of patients are presented in Table I. In addition, 126 healthy individuals of both sexes $(M / F=63 / 63)$ served as a control group for the SNP study that involved 132 patients. Research was carried out in compliance with the Helsinki Declaration. The approval of Bioethical Committee of Wroclaw Medical University was obtained for the study. Informed consent was obtained from all individual participants included in the study.

Immunomodulating therapy and response.

Thalidomide. Ninety-four patients were treated with thalidomidecontaining regimens $(81 \%$ with CTD: cyclophosphamide thalidomide, dexamethasone). Response to first line therapy in patients was as follows: complete remission (CR) 14 patients, very good partial response (VGPR) 12 patients, partial response (PR) 41 patients, minor response (MR) 6 patients, stable disease (SD) 14 patients, progressive disease (PD) 7 patients. In 48 patients, megachemotherapy supported by autologous stem cell transplantation (autoBMT) was applied after first line therapy. Responses after autoBMT were as follows: 17 CR, 20 VGPR, 11 PR. Median progression free survival after induction chemotherapy was 14 months (range $=0-60$ months). We observed 20 cases of thalidomide-induced neuropathy and 2 cases were complicated by a thrombotic event while on thalidomide.

Lenalidomide. 27 patients out of the whole analyzed population were treated with lenalidomide-dexamethasone (Len-Dex) regimen, and for majority of them it was a third line therapy. Median number of cycles administered was 7 (range $=2-35$ cycles). In 17 patients neutropenia grade 6 was reported. Therapy was complicated by thrombosis in one patient. Responses to lenalidomide were as follows: 3 cases of CR, 6 cases of VGPR, 3 PR, 5 SD and 4 patients progressed while on lenalidomide. Median progression free survival was 5 months (range=3-31 months).
$V E G F$ and bFGF genotyping. Ninety-six and 132 patients were typed for $V E G F$ and $b F G F$ polymorphism, respectively. One hundred and twenty-three healthy individuals served as a control group. The $V E G F$ and $b F G F$ alleles were detected using a polymerase chain reaction restriction fragment length polymorphism (PCR-RFLP) assay as previously described $(8,9)$.

Statistical analysis. Genotype and allele frequencies were compared between the study groups by the Fisher's exact test. The odds ratio (OR) was calculated by Haldane's modification of Woolf's method and the significance of its deviation from unity was estimated by Fisher's exact test. Survival analyses were performed employing Kaplan-Maier analysis and log rank test. Probability values $p<0.05$ were considered statistically significant, and those between 0.05 and 0.1 as indicative of a trend.

\section{Results}

Distribution of alleles and genotypes of VEGF and bFGF encoding genes in multiple myeloma patients and controls. The $V E G F$ rs3025039 and $b F G F$ rs308395 alleles and genotypes segregated similarly in patients with multiple myeloma and healthy individuals. None of the polymorphisms studied thus appeared to be associated with susceptibility to the disease. The $V E G F$ CC homozygous genotype was the most frequent and was carried by over $80 \%$ of individuals in both groups tested, 83 out of $96(86.5 \%)$ patients and 101 out of $122(82.8 \%)$ healthy individuals. The VEGF $\mathrm{T}$ variant was present in 13 (3 homo-, 10 heterozygotes) out of 96 patients and 21 (including 8 homozygotes) out of 122 healthy individuals.

Among 132 multiple myeloma patients genotyped for the $b F G F$ alleles, 99 (75.0\%) patients were $C C$ homozygotes, 31 $(23.5 \%)$ were heterozygous for the $b F G F$ alleles, and 2 $(1.5 \%)$ were carrying the $G G$ homozygous genotype. Ninetynine (80.5\%), $23(18.7 \%)$ and $1(0.8 \%)$ out of 123 healthy individuals were carrying the $b F G F C C, G C$ and $G G$ genotypes, respectively. The minor allelic frequencies were as follows: for the VEGF $936 T$ it equalled 0.083 and 0.119 , for patients and controls, respectively, and for the $b F G F$ $921 G 0.133$ and 0.102 , respectively.

Distribution of the $V E G F$ and $b F G F$ alleles and genotypes in controls closely resembles that observed in other Caucasian populations (described by Beránek et al. (7), Galimberti et al. (10), Kariz et al. (11), Petrovic et al. (12)). The VEGF CC homozygous genotype was overrepresented among patients with multiple myeloma (86.5\%) as compared to patients with NHL $(78.2 \%, p=0.080)$ and patients with CLL $(70.6 \%, p=0.017)$ analyzed in our previous studies $(8$, 9). On the other hand, the frequencies of the $b F G F$ alleles and genotypes in multiple myeloma patients did not significantly differ from those previously observed in CLL and NHL patients.

Interestingly, it was found that patients presenting with stage I-II of the disease (according to the Durie-Salmon criteria) more frequently carried the $b F G F-921 G$ allele as 
Table I. Patient characteristics.

\begin{tabular}{lc}
\hline Data & $\mathrm{N}$ \\
\hline Gender $(\mathrm{n}=132)$ & \\
$\quad$ Male & 70 \\
Female & 62 \\
Age $(\mathrm{n}=120)$ & \\
$\quad \geq 50$ years & 102 \\
$\quad<50$ years & 18 \\
Stage according to Durie-Salmon criteria $(\mathrm{n}=117)$ & \\
I & 25 \\
II & 48 \\
III & 44 \\
International staging system (n=117) & \\
I & 39 \\
II & 42 \\
III & 36 \\
Myeloma type $(\mathrm{n}=119)$ & \\
IgG & 68 \\
IgA & 31 \\
Light chain disease $(\mathrm{LCD})$ & 15 \\
Non-secretory & 4 \\
Solitary & 1 \\
Beta-2-microglobulin (n=112) & 12 \\
$\quad \leq$ norm $(\leq 1.80 \mathrm{mg} / \mathrm{l})$ & 100 \\
$>$ norm $(>1.80 \mathrm{mg} / \mathrm{l})$ & 34 \\
Anaemia $(\mathrm{n}=114)$ & 80 \\
$\quad<10$ g/dl & \\
$\geq 10$ g/dl & \\
\hline
\end{tabular}

compared to patients in stage III multiple myeloma and healthy controls (Figure 1A). Among 73 patients in stage III, 24 were carrying the $\mathrm{G}$ allele as compared to 7 out of 44 patients in stage III $(0.33$ vs. $0.16, p=0.053)$ and 24 out of 123 healthy individuals $(\mathrm{OR}=2.010, p=0.040)$. Moreover, the $b F G F-913 G$ allele was observed more frequently in patients with lambda light chains of $\operatorname{IgA}$ and $\operatorname{IgG}$ than in those with kappa chains $(13 / 30$ vs. $13 / 69, p=0.014)$.

Genetic variability within the VEGF and bFGF genes and response to immunomodulating therapy. The presence of the $b F G F-921 G$ allele was found to be associated with worse response to immunomodulating therapy. Progression of the disease after first line therapy was more frequent among patients carrying this allelic variant $(6 / 32 v s .4 / 88, p=0.022$, Figure 1B). Similar relationships were seen in subgroups of patients stratified with respect to the chemotherapeutic regimens used, although not all of these relationships reached statistical significance.

In patients treated with the CTD regimen (cyclophosphamide, thalidomide, dexamethasone), presence of the $b F G F-921 G$ allele tended to be more frequent among those whose disease progressed after therapy (4/20 vs. 3/56,
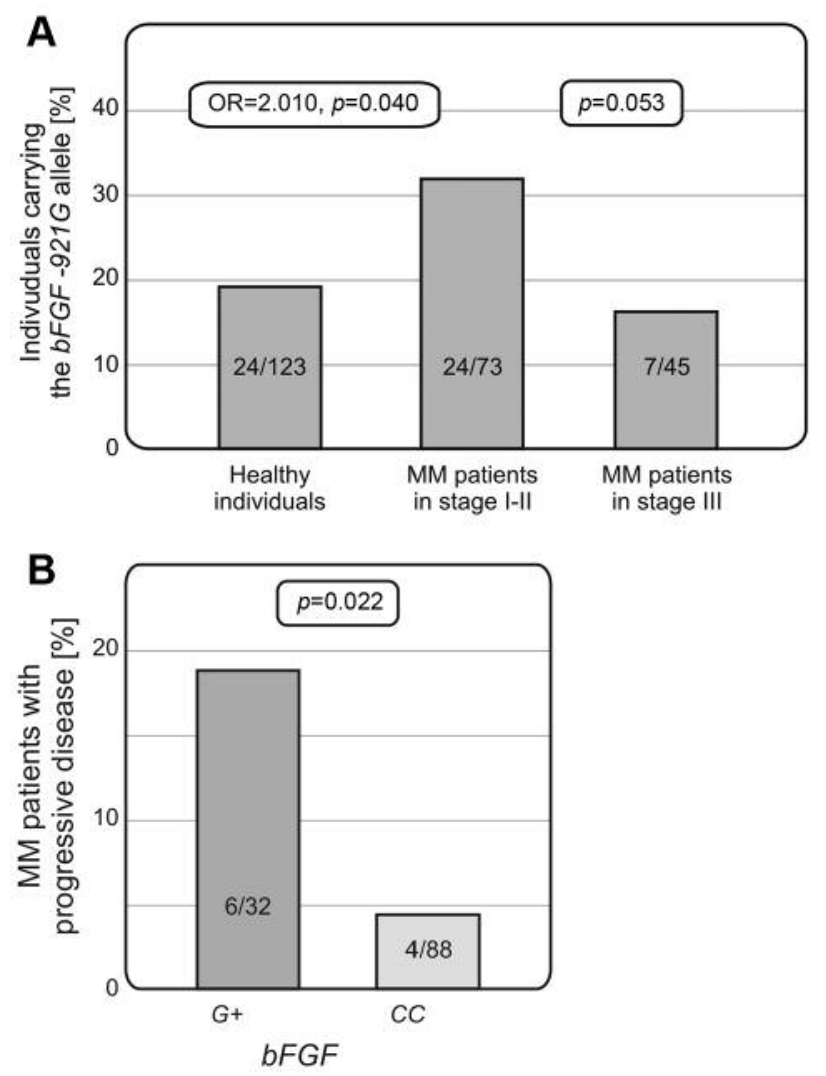

Figure 1. Associations of the bFGF (rs308395; $-921 \quad C>G)$ polymorphism with disease severity $(A)$ and response to therapy $(B)$. Stage III of multiple myeloma and progression of the disease after first line chemotherapy is more frequent among patients carrying the bFGF $-921 G$ allelic variant.

$p=0.073)$ and in the group treated with MP/MPT/MPV (Mmelphalan, P-prednisone, T-thalidomide, V-bortezomib) there were more individuals positive for the bFGF $\mathrm{G}$ allele among those who poorly responded to the administered therapy (stable or progressive disease; $2 / 4$ vs. $0 / 12, p=0.050$ ) compared to those with CR, VGPR or PR. We also observed that among patients treated with thalidomide (alone or in combination with other agents), the $b F G F-921 G$ allele was more common in patients who progressed after treatment (4/24 vs. 3/69, $p=0.070$ ). Although the above-mentioned relationships are not considered significant, they might indicate that the therapy with thalidomide is affected by the bFGF polymorphism.

The effect of the $b F G F$ polymorphism on the lenalidomide therapy was also assessed. Among 27 patients subjected to lenalidomide-dexamethasone (Len-Dex) regimen, 8 were carrying the $b F G F C G$ heterozygous genotype, while 19 were wild type homozygotes (with the $\mathrm{CC}$ genotype). Treatment related neutropenia was observed among 3 out of 6 patients with the $G$ allele and only in 4 out of $11 C C$ homozygotes 
(0.50 vs. 0.27, ns). Patients carrying the $b F G F-921 G$ allele responded less favourably to Len-Dex regimen. Progression of the disease after treatment was more frequent among carriers of the $b F G F G$ allele (2/4 vs. $4 / 17, \mathrm{~ns})$.

No significant association was observed for the $V E G F$ polymorphism and response to treatment. No relationship was detected between polymorphic variants of the $V E G F$ and $b F G F$ genes and survival.

\section{Discussion}

The present study aimed to assess the potential association between the VEGF (rs3025039; $936 \mathrm{C}>\mathrm{T})$ and $b F G F$ (rs308395; -921 C>G) polymorphisms and the risk of multiple myeloma. None of the polymorphisms studied was found to the associated with susceptibility to the disease. However, presence of the $b F G F C C$ wild-type genotype appeared to predispose patients to develop more advanced (stage III according to the Durie-Salmon's criteria) disease. This relationship has not been previously described.

Simiraly to our results, a previous study by Faber et al. (13) showed that there are no associations between the $V E G F$ polymorphism and multiple myeloma risk. However, in their study, the VEGF 936CC wild type homozygosity contributed to the risk of multiple myeloma in combination with either the GSTM1 undeleted genotype or GSTM1 undeleted + GSTT1 null combined genotypes. Both these genes, the GSTM1 and the GSTT1, encode the glutathione Stransferase (GST) enzymes. Thus, is seems that there is no direct association of the VEGF (rs3025039; $936 \mathrm{C}>\mathrm{T})$ single nucleotide polymorphism (SNP) with the predisposition to multiple myeloma, although other SNPs within the VEGF gene have been implicated in multiple myeloma (14). Brito et al. found the higher frequencies of the $V E G F-2578 C C$ homozygosity and -2578CC/-634GG, -2578CC/-1154GG/$634 G G$ VEGF genotype combinations in patients than in controls, associated with a 1.89-, a 5.52-, and a 4.91-fold increased risk for multiple myeloma, respectively. Moreover, they also observed that VEGFR2 polymorphism may contribute to the risk of multiple myeloma. In their study $V E G F-2578 C C$ plus VEGFR2 + 1192GG, VEGF -2578CC plus VEGF -634GG plus VEGFR2 + 1192GG, and VEGF $1154 G G$ plus VEGF $-634 G G$ plus VEGFR2 -604TT combined genotypes were more frequently detected in patients than healthy individuals.

Furthermore, having analyzed 4 SNPs within the VEGF gene $(-2578 \mathrm{C}>\mathrm{A}(\mathrm{rs} 699947),-460 \mathrm{C}>\mathrm{T}(\mathrm{rs} 833061),+405 \mathrm{G}>\mathrm{C}$ (rs2010963) and +936C > T (rs3025039)), Andersen et al. (15) reported a possible relation between the haplotype -2578A/$460 C /+405 G(A C G)$ and the effects of thalidomide treatment. Patients with no copies of the $A C G$ haplotype had a significantly longer time to next treatment than patients with one or two copies of this haplotype. However, no association with the +936C>T (rs3025039) SNP was observed in that study, which is in agreement with our results.

There have been a number of studies that aimed to assess the effect of various gene polymorphisms on the therapy of patients with multiple myeloma (summarized in Vangsted et al. (16)) These included analyses of several SNPs in genes encoding molecules involved in DNA repair, apoptosis, inflammation or function of the nervous system. The influence of cytochrome $P 4502 \mathrm{C} 19$ gene variations has also been studied and the effect was seen in Asian populations but not in Europeans (17-19).

To our knowledge, none of the previous studies considered the role of SNPs within the $b F G F$ gene in the response to treatment in patients with multiple myeloma. We found that the $b F G F-921 \mathrm{C}>\mathrm{G}$ (rs308395) SNP might be of prognostic value for the patients with multiple myeloma treated with thalidomide, although our results did not reach statistical significance on this. It should, however, be noted that an earlier study showed that thalidomide and bFGF interact somehow, probably indirectly, as the bFGF-induced cell proliferation and VEGF expression were reduced after thalidomide treatment (20). Also of note, a recent study showed that expression of VEGF and bFGF was significantly different in patients responding to IMiD-based therapy than in those who did not respond to that therapy (21). We observed that progressive disease was more frequent in patients carrying the $b F G F-921 \mathrm{G}$ allele.

These results suggest that the $b F G F \mathrm{G}$ allele might be a potential marker associated with worse response to therapy in patients with multiple myeloma. However, these data need to be verified in more extended studies involving numerous groups of patients.

\section{Conflicts of Interest}

The Authors declare no conflicts of interest.

\section{Acknowledgements}

This work was supported by statutory activity of Wroclaw Medical University (Project No. ST-370).

\section{References}

1 de la Puente P, Muz B, Azab F and Azab AK: Cell trafficking of endothelial progenitor cells in tumor progression. Clin Cancer Res 19: 3360-3368, 2013.

2 Vacca A and Ribatti D: Bone marrow angiogenesis in multiple myeloma. Leukemia 20: 193-199, 2006.

3 de la Puente P, Muz B, Azab F, Luderer M and Azab AK: Molecularly targeted therapies in multiple myeloma. Leuk Res Treatment 2014: 976567, 2014.

4 Vincenti V, Cassano C, Rocchi M and Persico G: Assignment of the vascular endothelial growth factor gene to human chromosome 6p21.3. Circulation 93: 1493-1495, 1996. 
5 Renner W, Kotschan S, Hoffmann C, Obermayer-Pietsch B and Pilger E: A common 936C/T mutation in the gene for vascular endothelial growth factor is associated with vascular endothelial growth factor plasma levels. J Vasc Res 37: 443-448, 2000.

6 Lafage-Pochitaloff M, Galland F, Simonetti J, Prats H, Mattei MG and Birnbaum D: The human basic fibroblast growth factor gene is located on the long arm of chromosome 4 at bands q26q27. Oncogene Res 5: 241-244, 1990.

7 Beránek M, Tschöplová S, Kanková K, Kuhrová V and Vácha $\mathrm{J}$ : Genetic variation in the promoter region of the basic fibroblast growth factor gene. Hum Immunol 64: 374-377, 2003.

8 Wróbel T, Mazur G, Dzietczenia J, Gebura K, Kuliczkowski K and Bogunia-Kubik K: VEGF and bFGF gene polymorphisms in Polish patients with B-CLL. Med Oncol 30: 456, 2013.

9 Wróbel T, Mazur G, Dzietczenia J, Gębura K, Kuliczkowski K and Bogunia-Kubik K: VEGF and bFGF gene polymorphisms in patients with non-Hodgkin's lymphoma. Biomed Res Int 2013: 159813, 2013.

10 Galimberti S, Nagy B, Palumbo GA, Ciancia E, Buda G, Orciuolo E, Melosi A, Lambelet P, Ronca F and Petrini M: Vascular endothelial growth factor polymorphisms in mantle cell lymphoma. Acta Haematol 123: 91-95, 2010.

11 Kariz S, Grabar D, Krkovic M, Osredkar J and Petrovic D: Polymorphisms in the promoter region of the basic fibroblast growth factor gene are not associated with myocardial infarction in a Slovene population with type 2 diabetes. J Int Med Res 37: 1596-1603, 2009.

12 Petrovic MG, Krkovic M, Osredkar J, Hawlina M and Petrovic D: Polymorphisms in the promoter region of the basic fibroblast growth factor gene and proliferative diabetic retinopathy in Caucasians with type 2 diabetes. Clin Experiment Ophthalmol 36: 168-172, 2008.

13 Faber EW, Lourenço GJ, Ortega MM, Lorand-Metze I, De Souza CA and Lima CS: Polymorphisms of VEGF, GSTM1 and GSTT1 genes in multiple myeloma risk. Hematol Oncol 30: 105-107, 2012.

14 Brito AB, Lourenço GJ, Oliveira GB, De Souza CA, Vassallo J and Lima CS: Associations of VEGF and VEGFR2 polymorphisms with increased risk and aggressiveness of multiple myeloma. Ann Hematol 93: 1363-1369, 2014.
15 Andersen NF, Vogel U, Klausen TW, Gimsing P, Gregersen H, Abildgaard $\mathrm{N}$ and Vangsted AJ: Vascular endothelial growth factor (VEGF) gene polymorphisms may influence the efficacy of thalidomide in multiple myeloma. Int J Cancer 131: E636E642, 2012.

16 Vangsted A, Klausen TW and Vogel U: Genetic variations in multiple myeloma II: association with effect of treatment. Eur J Haematol 88: 93-117, 2012.

17 Li Y, Hou J, Jiang H, Wang D, Fu W, Yuan Z, Chen Y and Zhou L: Polymorphisms of CYP2C19 gene are associated with the efficacy of thalidomide based regimens in multiple myeloma. Haematologica 92: 1246-1249, 2007.

18 Matsuzawa N, Nakamura K, Matsuda M, Ishida F and Ohmori S: Influence of cytochrome P450 2C19 gene variations on pharmacokinetic parameters of thalidomide in Japanese patients. Biol Pharm Bull 35: 317-320, 2012.

19 Vangsted AJ, Søeby K, Klausen TW, Abildgaard N, Andersen NF, Gimsing P, Gregersen H, Vogel U, Werge T and Rasmussen HB: No influence of the polymorphisms CYP2C19 and CYP2D6 on the efficacy of cyclophosphamide, thalidomide, and bortezomib in patients with multiple myeloma. BMC Cancer 10: 404, 2010.

20 Tan H, Chen H, Xu C, Ge Z, Gao Y, Fang J, Liu W and Xiao S: Role of vascular endothelial growth factor in angiodysplasia: an interventional study with thalidomide. J Gastroenterol Hepatol 27: 1094-1101, 2012.

21 Medinger M, Halter J, Heim D, Buser A, Gerull S, Stern M and Passweg J: Angiogenic markers in plasma cell myeloma patients treated with novel agents. Anticancer Res 35: 1085-1090, 2015.

Received December 19, 2016

Revised March 8, 2017

Accepted March 9, 2017 